\title{
Nützt Brachytherapie älteren Frauen mit Mammakarzinom?
}

Weil sie unter anderem schonender als eine Bestrahlung der gesamten Brust und zudem weniger aufwendig ist, wird beim Mammakarzinom immer öfter die Brachytherapie angewandt. Bei Frauen, die älter als 67 Jahre sind, sieht das Ergebnis aber nicht so ermutigend aus, wie die Ergebnisse einer retrospektiven US-Studie mit fast 93.000 Patientinnen vermuten lassen.

$\mathrm{N}$ ach Angaben der Deutschen Gesellschaft für Radioonkologie ist die Brachytherapie integraler Bestandteil der Radioonkologie und wird von der Gesellschaft so gefördert, dass sie flächendeckend angeboten werden kann. Der Anteil der älteren Patientinnen mit Brustkrebs, bei denen eine Brachytherapie vorgenommen wird, liegt weltweit bei $10 \%$.

Den Empfehlungen der Europäischen Gesellschaft für Brachytherapie zufolge kann das Verfahren nach einer Operation u. a. dann angewendet werden, wenn die Patientinnen älter als 60 Jahre sind und der Tumor nicht größer als $2 \mathrm{~cm}$ ist. In der US-Studie wurden nun die Daten von Patientinnen des US-Medicare-Programms mit Einzelleistungsvergütung analysiert. Die Frauen waren mindestens 67 Jahre alt. Nach der Lumpektomie erhielten 6.952 von ihnen eine Brachytherapie und 85.783 eine Bestrahlung der gesamten Brust.

Die Patientinnen mit Brachytherapie schnitten schlechter ab. Die Inzidenz einer Mastektomie nach fünf Jahren in der Brachytherapie-Gruppe lag bei 3,95\%, in der Vergleichsgruppe bei 2,18\% - ein signifikanter Unterschied. Waren die axillären Lymphknoten befallen, dann war der Unterschied mit 8,25 versus 2,53\% sogar noch größer.

Infektionen waren mit 16,2 vs. $10,33 \%$ signifikant häufiger unter der Brachytherapie als bei der Ganzbrustbestrahlung. Auch traten bei Patientinnen mit Teilbrustbestrahlung mit 16,25 vs. $9 \%$ signifikant häufiger nicht infektiöse Komplikationen auf. Das Gesamtüberleben unterschied sich nicht signifikant: Nach fünf Jahren lebten in der Gruppe mit Brachytherapie noch fast $88 \%$ der Patientinnen, in der Vergleichsgruppe $87 \%$.

An der Vergleichsstudie nahm nur eine ausgewählte Gruppe von Patientinnen teil. Den Autoren zufolge müssten die beiden Therapieformen in künftigen Studien auch bei jüngeren Patientinnen mit anderen Versicherungsarten verglichen werden. Solche Studien müssten zudem eine deutlich längere Nachbeobachtungsdauer als die aktuelle Studie haben.

Den Nutzen der einen oder anderen Bestrahlungsart soll nun die Phase-IIIStudie NSABP B 39/RTOG 0413 des „National Surgical Adjuvant Breast and Bowel Project“ und der „Radiation Therapy Oncology Group" klären. Darin sollen bis Januar 2013 4.300 Patientinnen mit Brustkrebs jünger als 50 Jahre aufgenommen werden, um die Wirksamkeit einer partiellen - als Brachytherapie oder externe Bestrahlung - mit der einer konventionellen Bestrahlung der gesamten Brust zu vergleichen (www.nsabp.pitt.edu). Eine endgültige Analyse der Daten wird in zehn Jahren erwartet. Peter Leiner

Smith GL et al. Association between treatment with brachytherapy vs whole-breast irradiation and subsequent mastectomy, complications, and survival among older women with invasive breast cancer. JAMA.

2012;307(17):1827-37.

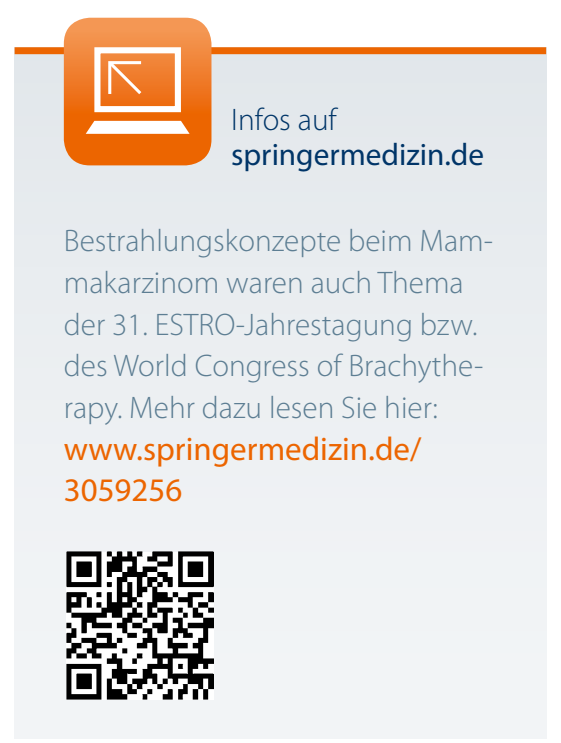

